\title{
Cross-polarization of electromagnetic waves reflected by anisotropic gradient planar structures
}

\author{
Natalja Moiseeva ${ }^{1, *}$ \\ ${ }^{1}$ Department of Radiophysics, IMIT, Volgograd State University, Volgograd, Russia
}

\begin{abstract}
The results of calculating the moduli of the coefficients of the electromagnetic wave reflection matrix (EMW) at the boundary of an inhomogeneous anisotropic medium with torsion using the matrix form of the Wentzel-Kramers-Brillouin (WKB) method are presented. The effect of the orientation of the optical axis and the torsion angle on the polarization of the waves propagating in the medium and on the off-diagonal coefficients of the reflection matrix is shown.
\end{abstract}

\section{Introduction}

Currently, there is an intensive development of optoelectronics and nanophotonics. For the development of new highly efficient optoelectronic devices: solar cells, nanodirectional devices, sensing, imaging, ultra-high resolution [1] materials that have unusual properties are used. The use of alloying additives in plasmonics opens up possibilities for the creation of metamaterials with controlled optical topology [2-3]. The need for modern computing increases the demand for high-speed optical devices, which are photonic analogues of electronic devices [4]. The possibilities of using multilayer heterostructures are not exhausted; Prospects for their application are listed in [5]. Liquid crystals are often used as materials for the new elemental base of photonics. The variety of their properties and variability in the application of external fields [6] allow them to be used for new optical solutions. Liquid crystals are used for fast optical switches [7], tunable color filters [8].

To calculate the fields in planar structures, researchers traditionally use a model of a layered medium. In this model, an inhomogeneous layer is represented as a set of layers with uniform parameters. Such a simplification leads to the fact that artificial discontinuities of the projections of the vectors of the fields of the electromagnetic wave at the interfaces of homogeneous layers appear in the problem, reflections at nonexistent boundaries are taken into account; this leads to computational interference. The WentzelKramers-Brillouin (WKB) method [9] allows us to find a solution for a medium with gradient parameters [10]. Useful approximate solutions obtained using the WKB method were found in [11], and the accuracy of the WKB solution was estimated in [12, 13]. In the vicinity of turning points, the method has a limitation, since the solutions of Wenzel, Kramers, and Brillouin diverge in the vicinity of turning points. The use of modified Airy

*Corresponding author: moiseeva@,volsu.ru 
functions avoids discontinuous solutions [14]. A rigorous method based on the Airy functions, designed to calculate the eigenvalues of optical waveguides with arbitrary index profiles in the presence of turning points, was proposed in [15]. A solution based on the WKB method was presented in [16], a $4 \times 4$ matrix form for the WKB method was obtained in [17]. In this work, the $4 \times 4$ matrix form for the Wenzel-Kramers-Brillouin method is used to calculate the coefficients of the light reflection matrix at the boundary of an inhomogeneous anisotropic liquid crystal with a variable direction of the optical axis.

\section{Formulation of the problem}

\subsection{Optical properties of an anisotropic medium}

Using the methods of classical electrodynamics, we study the problem of light reflection at the boundary of an inhomogeneous anisotropic layer of a cholesteric liquid crystal (CLC) with dispersion at an inclined incidence. Figure 1 shows an inhomogeneous anisotropic layer of a liquid crystal with the direction of the optical axis varying along $0 \mathrm{Z}$.

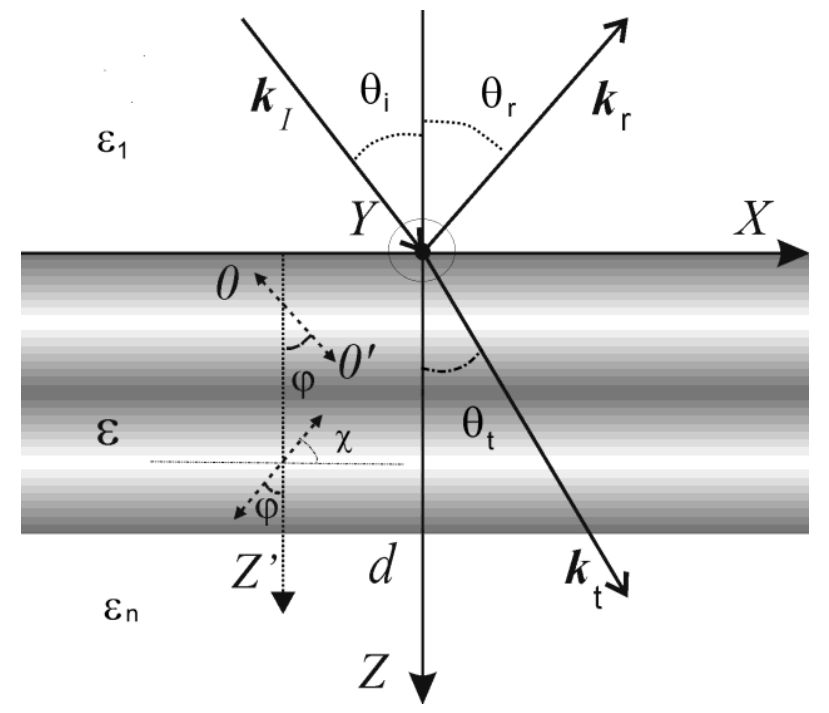

Fig. 1. The plane of incidence of light on an anisotropic inhomogeneous layer with torsion of the optical axis. Angle of incidence is $\theta_{i}$.

Dielectric constant tensor for CLC has the form:

$$
\boldsymbol{\varepsilon}(\omega, z)=\left(\begin{array}{ccc}
\varepsilon_{o}(\omega) \cos ^{2}(\chi(z))+\varepsilon_{e} \sin ^{2}(\chi(z)) & \frac{\varepsilon_{e}(\omega)-\varepsilon_{0}(\omega)}{2} \sin (2 \chi(z)) & 0 \\
\frac{\varepsilon_{e}(\omega)-\varepsilon_{0}(\omega)}{2} \sin (2 \chi(z)) & \varepsilon_{o}(\omega) \sin ^{2}(\chi(z))+\varepsilon_{e}(\omega) \cos ^{2}(\chi(z)) & 0 \\
0 & 0 & \varepsilon_{o}(\omega)
\end{array}\right)
$$

The dependence of $\varepsilon_{o}$ and $\varepsilon_{e}$ on the frequency $\omega$ is written as:

$$
\varepsilon_{o, e}(\omega)=\varepsilon_{\perp, \|}+\frac{\omega_{P}^{2} F_{\perp, \|}}{\omega_{0}^{2}-\omega^{2}-i \omega \gamma_{\perp, \|}} .
$$


where $\lambda_{0}=9.07 \mu \mathrm{m}, \alpha_{\perp}=0.076 \mu m^{-1}, \alpha_{\|}=0.075 \mu m^{-1}$, where $\alpha_{\perp}$ and $\alpha_{\|}$- absorption for waves s- and p- polarization, $\mathrm{F}_{\perp}=0.1, \mathrm{~F}_{\|}=0.05 \quad$ [18], $\varepsilon_{\perp}=5.17, \varepsilon_{\|}=4.58$ [19], $\gamma_{12}=0,5\left(\gamma_{\perp}+\gamma_{\|}\right)$. For given parameter values, we obtain: $\gamma_{\perp}=8.017 \cdot 10^{-3} \cdot \omega_{0}$, $\gamma_{\|}=4.316 \cdot 10^{-3} \cdot \omega_{0}$. Layer thickness $d=15 \lambda_{0}$. The optical axis is parallel to the interface, its direction changes with distance from the boundary: $\chi(z)=\chi_{0}+\mathrm{K} \cdot z$. Consider the case when $\mu$ - is a scalar. The substrate is glass type $\mathrm{K} 8$.

\subsection{Basic equations}

From the Maxwell equations for field rotors $\mathbf{E}$ and $\mathbf{H}$

$$
\begin{aligned}
& \operatorname{rot} \mathbf{E}=-\frac{1}{c} \frac{\partial \mathbf{B}}{\partial t}, \\
& \operatorname{rot} \mathbf{H}=\frac{1}{c} \frac{\partial \mathbf{D}}{\partial t} .
\end{aligned}
$$

The material equations $\mathbf{B}=\mu \mathbf{H}$ and $\mathbf{D}=\boldsymbol{\varepsilon} \mathbf{E}$, for the case of oblique incidence follows a system of ordinary differential equations

Or, briefly:

$$
\frac{d}{d z}\left(\begin{array}{c}
E_{Y} \\
H_{X} \\
H_{Y} \\
E_{X}
\end{array}\right)=i k_{0}\left(\begin{array}{cccc}
0 & -\mu & 0 & 0 \\
-\left(\frac{\Delta \varepsilon_{22}}{\varepsilon_{33}}-\frac{\alpha^{2}}{\mu}\right) & 0 & \alpha \frac{\varepsilon_{23}}{\varepsilon_{33}} & -\frac{\Delta \varepsilon_{21}}{\varepsilon_{33}} \\
\frac{\Delta \varepsilon_{12}}{\varepsilon_{33}} & 0 & \frac{\alpha \varepsilon_{13}}{\varepsilon_{33}} & \frac{\Delta \varepsilon_{11}}{\varepsilon_{33}} \\
-\alpha \frac{\varepsilon_{32}}{\varepsilon_{33}} & 0 & \mu-\frac{\alpha^{2}}{\varepsilon_{33}} & \frac{\alpha \varepsilon_{13}}{\varepsilon_{33}}
\end{array}\right)\left(\begin{array}{c}
E_{Y} \\
H_{X} \\
H_{Y} \\
E_{X}
\end{array}\right) .
$$

$$
\frac{d \mathbf{Q}}{d z}=i k_{0} \mathbf{A Q}
$$

Besignations that were used $\alpha=\frac{k_{\|}}{k_{0}}=n(z) \sin \theta(z)=$ const, according to Snell's law, $\Delta \varepsilon_{11}=\varepsilon_{11} \varepsilon_{33}-\varepsilon_{13} \varepsilon_{31}, \quad \Delta \varepsilon_{22}=\varepsilon_{22} \varepsilon_{33}-\varepsilon_{23} \varepsilon_{32}, \quad \Delta \varepsilon_{12}=\varepsilon_{12} \varepsilon_{33}-\varepsilon_{13} \varepsilon_{32}$. The fundamental matrix of the solution is obtained, of the form [20]:

$$
\mathbf{Y}(z)=\mathbf{F} \cdot \operatorname{diag}\left[e^{i k_{0} \int_{0}^{z} \lambda_{1}(\xi) d \xi}, \ldots, e^{i k_{0} \int_{0}^{z} \lambda_{4}(\xi) d \xi}\right] .
$$

$\lambda_{i}$ - eigenvalues of the matrix $\mathbf{A}$ of system (4):

$$
\operatorname{det}(\mathbf{A}-\lambda \mathbf{I})=0
$$

the matrix $\mathbf{F}$ was obtained by substituting $\mathbf{Y}(z)$ into system (4). The calculation of the tensor $\boldsymbol{\varepsilon}(\omega, z)$ showed that when light falls from the first medium - air, a solution of the form (5) will not have discontinuities $\operatorname{Re}\left(\varepsilon_{o, e}-\alpha^{2}\right)>0$, since there will be no turning points in the whole layer. The Cauchy matrix of the ODE system (4) has the form:

$$
\mathbf{N}(z, 0)=\mathbf{Y}(z) \mathbf{Y}^{-1}(0) \text {. }
$$




\section{Reflection matrix}

The matrix solution (6) allows us to calculate the field projections in an arbitrary $z=$ const plane [21]:

$$
\mathbf{Q}(z)=\mathbf{N}(z, 0) \mathbf{Q}(0) .
$$

For vectors $\mathbf{Q}_{i}, \mathbf{Q}_{r}, \mathbf{Q}_{t}$, composed of the tangential projections of the incident, reflected and transmitted waves, the matching of the boundary conditions in matrix form has the form:

$$
\mathbf{Q}_{t}(z)=\mathbf{N}(z, 0)\left(\mathbf{Q}_{i}(z)+\mathbf{Q}_{r}(z)\right)
$$

where

$$
\mathbf{N}(z, 0)=\left(\begin{array}{llll}
n_{11}(z, 0) & n_{12}(z, 0) & n_{13}(z, 0) & n_{14}(z, 0) \\
n_{21}(z, 0) & n_{22}(z, 0) & n_{23}(z, 0) & n_{24}(z, 0) \\
n_{31}(z, 0) & n_{32}(z, 0) & n_{33}(z, 0) & n_{34}(z, 0) \\
n_{41}(z, 0) & n_{42}(z, 0) & n_{43}(z, 0) & n_{44}(z, 0)
\end{array}\right) .
$$

From formula (8a) it follows:

$$
\left(\begin{array}{ll}
s_{11} & s_{12} \\
s_{21} & s_{22}
\end{array}\right)\left(\begin{array}{c}
E_{s i} \\
E_{p i}
\end{array}\right)=\left(\begin{array}{ll}
x_{11} & x_{12} \\
x_{21} & x_{22}
\end{array}\right)\left(\begin{array}{c}
E_{s r} \\
E_{p r}
\end{array}\right) .
$$

The reflection matrix has the form:

$$
\mathbf{R}=\left(\begin{array}{ll}
R_{s s} & R_{s p} \\
R_{p s} & R_{p p}
\end{array}\right)=\mathbf{X}^{-\mathbf{1}} \mathbf{S} .
$$

The coefficients of the reflection matrix (11) are complex. In the present work, the angular spectra of the absolute values of these coefficients were calculated: $\left|R_{s s}\right|,\left|R_{s p}\right|,\left|R_{p s}\right|,\left|R_{p p}\right|$ when the frequency changes near the resonance $\omega_{0}: \omega=\omega_{0}+\Delta \omega$. The torsion angle at the boundary $\mathrm{z}=0$ is $\chi_{0}=45^{\circ}$, at the boundary $\mathrm{z}=\mathrm{d}: \chi(d)=135^{\circ}$.

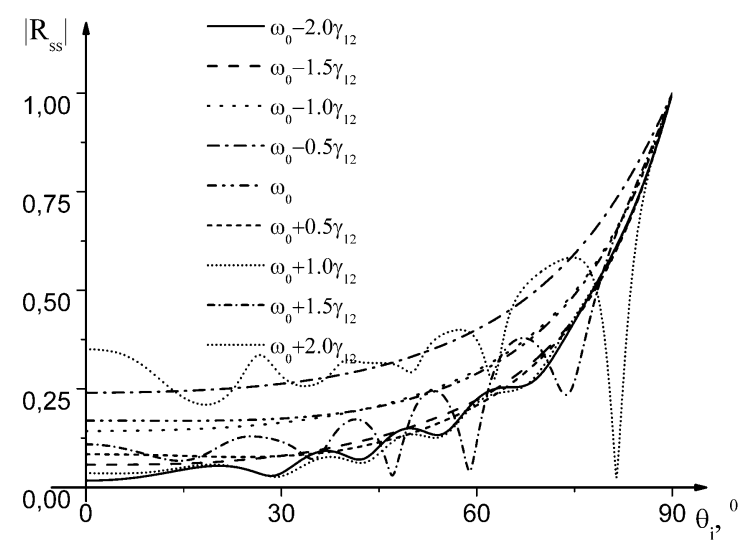

Fig. 2. Angular dependence of the modulus of the amplitude reflection coefficient $R_{s s}$ in the vicinity of the resonant frequency

The calculation showed the dependence of the coefficients $R_{s p}$ and $R_{p s}$ on the torsion angle $\Delta \chi$. When $\Delta \chi$, is a multiple of $\pi$, the coefficients $R_{s p}$ and $R_{p s}$ turn to zero. Figures 5 and 6 show the dependences of the absolute values of the coefficients $R_{s p}$ и $R_{p s}$ with a change in the angle of incidence and the angle $\Delta \chi$ in the interval $\left[\begin{array}{ll}0, & 90^{\circ}\end{array}\right]$. The 
calculation showed that with a change in the angle of rotation of the optical axis, it is possible to control the cross-polarization of light and use liquid crystals to modulate optical signals.

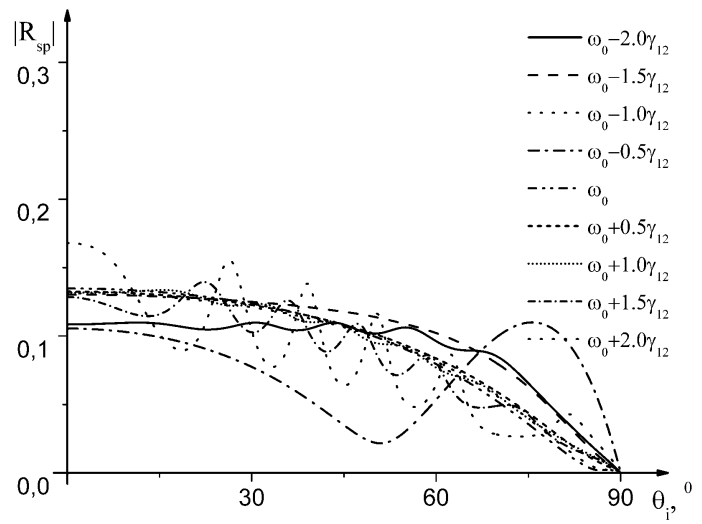

Fig. 3. Angular dependence of the modulus of the amplitude reflection coefficient $R_{\text {sp }}$ in the vicinity of the resonant frequency

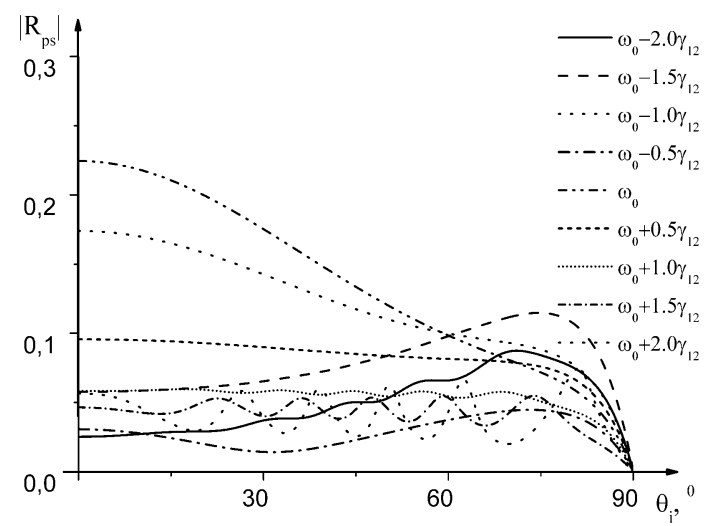

Fig. 4. Angular dependence of the modulus of the amplitude reflection coefficient $R_{s p}$ in the vicinity of the resonant frequency

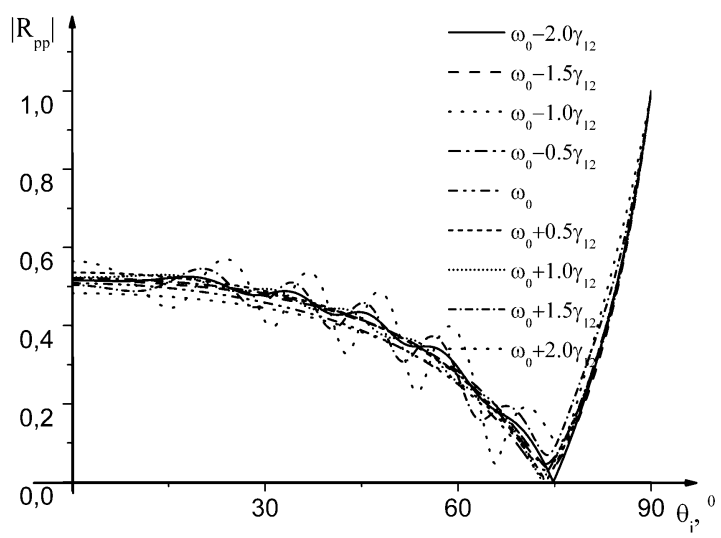

Fig. 5. Angular dependence of the modulus of the amplitude reflection coefficient $R_{p p}$ on the angle of incidence in the vicinity of the resonant frequency. 


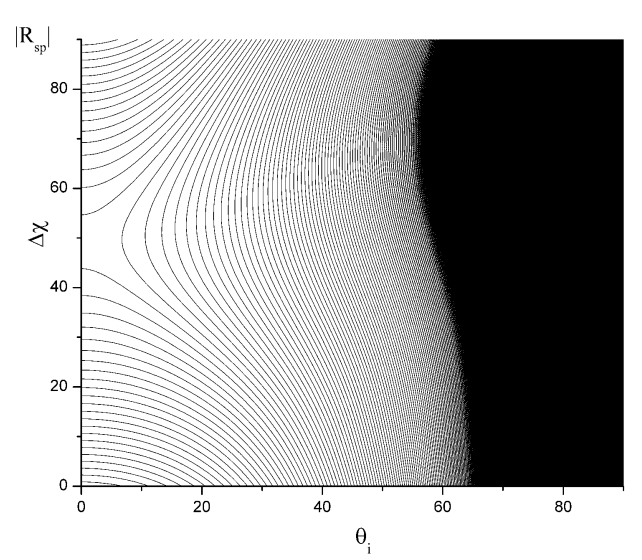

Fig. 6. The dependence of the modulus of the amplitude reflection coefficient $\mathrm{R}_{\mathrm{sp}}$ on the angle of incidence $\theta_{i}$ and the torsion angle $\Delta \chi$.

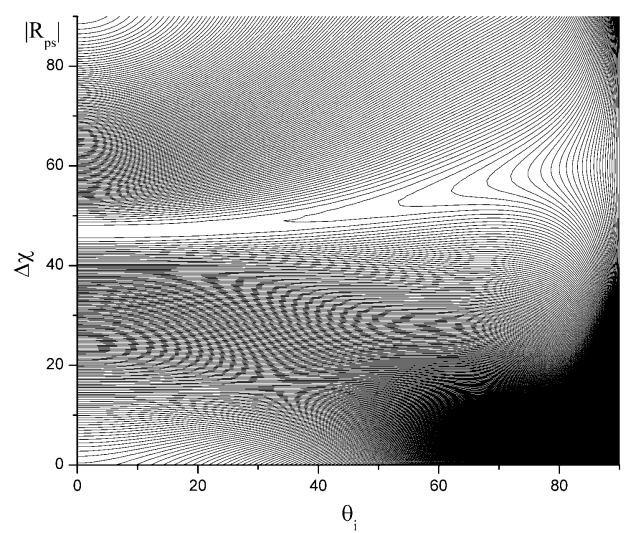

Fig. 7. The dependence of the modulus of the amplitude reflection coefficient $\mathrm{R}_{\mathrm{ps}}$ on the angle of incidence $\theta_{i}$ and the torsion angle $\Delta \chi$.

\section{Conclusions}

The dependences of the absolute values of the amplitude coefficients of the reflection matrix for the inhomogeneous anisotropic liquid crystal layer with dispersion and torsion of the optical axis were calculated using the $4 \times 4$ matrix form of the Wentzel- KramersBrillouin method. The dependence of the angular spectra of the coefficients of the reflection matrix on the torsion angle of the optical axis in the layer is shown.

\section{References}

1. O. Takayama, A. V. Lavrinenko J. Opt. Soc. Am. B, 36 F38-F48 (2019)

2. A. Dutta, D. Y. Wan, B. X. Yan, V. M. Shalaev, T. Venkatesan, A. Boltasseva CLEO (OSA) JW2A.63 (2019)

3. E. Simmons, K. Li, A. F. Briggs, S. R. Bank, D. Wasserman, E. Narimanov, V. A. Podolskiy CLEO FTh4M.5 (2019)

4. K. Srinivasan, B. J. H. Stadler Opt. Mater. Express 8, 3307-3318 (2018) 
5. R.Soref APL Photonics 3021101 (2018)

6. Y. J. Lim, J. H. Yoon, H. Yoo, S. M. Song, R. Manda, S. Pagidi, M.-H. Lee, J.-M. Myoung, S. H. Lee Opt. Mater. Express 8 3698-3707 (2018)

7. F. Jahanbakhsh, A. Lorenz, Appl. Opt. 58 5587-5594 (2019)

8. S. Chandrasekhar Liquid Crystals (Cambridge University Press, Cambridge, 1992)

9. Landau L. D., Lifshits E. M. Electrodynamics of Continuous Media (Pergamon Press, Oxford, 1984)

10. V. V. Zheleznyakov, V. V. Kocharovskii, V. V. Kocharovskii Sov. Phys. Usp. 26 877905

11. D. Marcuse in IEEE Journal of Quantum Electronics 9 1000-1006 (1973)

12. J. Janta, J. Čtyroký Optics Communications 25 49-52 (1978)

13. L. Lindblom, R. T. Robiscoe Journal of Mathematical Physics 32, 1254 (1991)

14. A.K. Ghatak, R.L. Gallawa, I.C. Goyal Modified Airy Function and WKB Solutions to the Wave Equation (NIST, Washington, 1991)

15. N. Zareian, P. Sarrafi, K. Mehrany and B. Rashidian, in IEEE Journal of Quantum Electronics 44 324-330 (2008)

16. F. Guo, L. Li, M. Wang SPIE/OSA/IEEE Asia Communications and Photonics (2007)

17. N. M. Moiseeva, A. V. Moiseev Proc. SPIE 107171071716 (2018)

18. S.-T. Wu Appl. Opt. 26 3434-3440 (1987)

19. S. M. Arakelyan, Y. S. Chilingaryan Nelinejnaya optika zhidkih kristallov (Nauka, Moscow, 1984)

20. W. Wasow Asymptotic Expansion for Ordinary Differential Equations (Dover publications, INC, Mineola, New York, 1965)

21. N. M. Moiseeva, A. V. Moiseev Computer Optics 42 354-361 (2018) 